\title{
Induction of Heme Oxygenase-1 by Sodium 9-Hydroxyltanshinone IIA Sulfonate Derivative Contributes to Inhibit LPS-Mediated Inflammatory Response in Macrophages
}

\author{
Xin-Hua Liu ${ }^{a}$ Xi-Ling Wang ${ }^{a}$ Hong Xin ${ }^{a}$ Dan Wu Xiao-Ming Xin ${ }^{a}$ Lei Miao \\ Qiu-Yan Zhang ${ }^{\text {a }}$ Yang Zhou $^{\text {b }}$ Qian Liu ${ }^{b}$ Qian Zhang ${ }^{\text {b }}$ Yi-Zhun Zhu ${ }^{a}$ \\ aShanghai Key Laboratory of Bioactive Small Molecules, Department of Pharmacology, School of \\ Pharmacy, Fudan University, Shanghai, 'b Department of Medicinal Chemistry, School of Pharmacy, \\ Fudan University, Shanghai, China
}

\section{Key Words}

Sodium 9-acetoxyltanshinone IIA sulfonate Inflammation $\bullet$ Heme oxygenase- 1 - Macrophage - Nuclear factor erythroid 2-related factor 2

\begin{abstract}
Background/Aim: Sodium 9-acetoxyltanshinone IIA sulfonate (ZY-1A4), a novel compound derived from sodium 9-hydroxyltanshinone IIA sulfonate, was synthesized with potential biological activities. This study aimed to explore the effects of ZY-1A4 on lipopolysaccharide (LPS)-triggered inflammatory response and the underlying mechanisms. Methods: Activation of RAW264.7 macrophages was induced by LPS. The effects of ZY-1A4 on inducible nitric oxide synthase (iNOS) expression, nitric oxide (NO) generation, nuclear factor-kB (NF-kB) activation, heme oxygenase-1 (HO-1) expression, and nuclear factor-erythroid 2 related factor 2 (Nrf2) pathway were evaluated to elucidate its underlying mechanisms on inflammatory responses. Results: ZY-1A4 concentration-dependently reduced iNOS expression and NO production, and inhibited c-Jun-N-terminal kinase $1 / 2$ (JNK1/2) phosphorylation and NF-KB activation in LPS-stimulated macrophages. In addition, ZY-1A4 concentration- and time-dependently induced $\mathrm{HO}-1$ expression associated with degradation of Kelch-like $\mathrm{ECH}$-associated protein 1 (Keap1) and nuclear translocation of Nrf2, while the effect of ZY-1A4 was abolished by a phosphoinositide 3-kinase (PI3K) inhibitor LY294002. Intriguingly, pharmacological inactivation of HO-1 with zinc protoporphyrin IX reversed anti-inflammatory effect of ZY$1 \mathrm{~A} 4$, but the anti-inflammatory effect of $\mathrm{ZY}-1 \mathrm{~A} 4$ was largely mimicked by $\mathrm{HO}-1$ by-products carbon monoxide and bilirubin. Furthermore, the inhibitory effect of ZY-1A4 on LPS-induced iNOS expression and NO release was abolished by HO-1 siRNA or LY294002. Conclusion: Our results demonstrated that ZY-1A4 suppressed LPS-induced iNOS expression and NO generation via modulation of NF-KB activation and $\mathrm{HO}-1$ expression. This new finding might shed light to the prevention and therapy of cardiovascular diseases.
\end{abstract}

Assist Prof. Qian Zhang

and Prof. Yi-Zhun Zhu

KARGER 125
Copyright (C) 2015 S. Karger AG, Basel

Department of Medicinal Chemistry, School of Pharmacy, Fudan University and Department of Pharmacology, School of Pharmacy, Fudan University, 826, Zhangheng Road, Pudong New District, Shanghai (China)

E-Mail Zhangqian511@shmu.edu.cn and E-Mail zhuyz@fudan.edu.cn 


\section{Introduction}

Inflammation not only protects host organisms from external injuries and pathogens, but also contributes to many pathophysiological conditions [1]. Macrophages are one of the critical immune cells in the regulation of inflammatory responses. Activated macrophages secrete a number of various inflammatory mediators, including nitric oxide (NO) and inducible nitric oxide synthase (iNOS) [2]. Excessive or unregulated production of these mediators has been implicated in mediating multiple inflammatory processes and cytotoxicity $[3,4]$. Transcription factor nuclear factor- $\kappa B(N F-\kappa B)$ is essential for LPS-induced iNOS expression and NO generation [5, 6]. In addition, several signaling molecules, such as phosphatidylinositol 3-kinase (PI3K)/protein kinase B (PKB/Akt) and mitogen-activated protein kinases (MAPK), also involve in LPS-mediated NF- $\mathrm{BB}$ activation and iNOS expression [7-10].

Cumulative evidences suggest heme oxygenase-1 (HO-1) functions as a cytoprotective enzyme against inflammatory responses through production of its metabolites such as carbon monoxide (CO), bilirubin, and free iron [11-14]. HO-1 also negatively regulates $N F-\kappa B$ activation and subsequent iNOS expression [15-17]. Transcription factor nuclear factor erythroid 2-related factor 2 (Nrf2) plays a predominant role in HO-1 expression [18]. Meanwhile, several signaling molecules, such as PI3K/Akt and MAPK, participate in the regulation of $\mathrm{HO}-1$ expression.

The Chinese medicinal herb Salvia miltiorrhiza Bunge (danshen) has been clinically used in Asian countries for the treatment of cardiovascular diseases [19, 20]. Recently, tanshinone IIA, one of bioactive constituents, has been shown to exert several pharmacological activities, including anti-oxidation, anti-inflammatory properties [19-21]. Owing to high lipophilicity of tanshinone IIA, sodium tanshinone IIA sulfonate (STS, Fig. 1A), a water-soluble derivative of tanshinone IIA, is synthesized and widely used for treatment of cardiovascular diseases $[16,20]$. However, various impurities are formatted in the process of storage or synthesis of STS [22, 23]. In our present study, an array of compounds are derived from sodium 9-hydroxyltanshinone IIA sulfonate, which is one of major impurities of STS during storage [23]. We conduct several biological evaluations of these compounds, and in the course of these evaluations, a novel derivative sodium 9-acetoxyltanshinone IIA sulfonate (ZY-1A4, Fig. 1B) is identified with significant anti-inflammatory activity. Therefore, we further conduct to investigate the effects of ZY-1A4 on LPS-induced inflammatory response in RAW264.7 macrophages and its underlying mechanisms.

Our results provide the first evidence that superinduction of HO-1 expression contribute to the novel compound ZY-1A4-mediated anti-inflammatory response in LPS-stimulated RAW264.7 cells, at least in part, through activation of Nrf2 and inhibition of NF- $\mathrm{\kappa B}$.

\section{Materials and Methods}

\section{Reagents and antibodies}

Dulbecco's modified Eagle's medium (DMEM) medium and fetal bovine serum (FBS) were from GIBCOBRL (USA). Wortmannin, LY294002, SP60012, and SB203580 were from Calbiochem (San Diego, CA). Antibodies against total- and phosphor (p) - c-Jun N-terminal kinase 1/2 (JNK1/2) ( $\mathrm{Thr}^{183}$ and Tyr $\left.{ }^{185}\right)$, totaland p- p38 $\left(\mathrm{Thr}^{180}\right.$ and $\left.\mathrm{Tyr}^{182}\right)$, total- and p- extracellular signal-regulated kinase 1/2 (ERK1/2) $\left(\mathrm{Thr}^{202} /\right.$ $\mathrm{Tyr}^{204}$ ), and NF- $\mathrm{KB}$ p65 were purchased from Cell Signaling Technology (Danvers, MA). Antibodies against $\beta$-actin, iNOS, keap1, and Nrf2 were obtained from Santa Cruz Biotechnology (Santa Cruz, CA). Antibody against Lamin A/C was from Epitomics (Burlingame, CA). LPS, CORM-3, bilirubin, zinc protoporphyrin IX (ZnPP), 3-(4, 5-dimetrylthiazol)-2, 5-diphenyltetrazolium bromide (MTT), and all other chemicals used in this study were purchased from Sigma-Aldrich (St. Louis, MO). ZY-1A4 was provided by Assist Prof. Qian Zhang (Department of Medicinal Chemistry, School of Pharmacy, Fudan University) and the purity was over 99\% determined by high performance liquid chromatography. ZY-1A4 was dissolved in dimethyl sulfoxide (DMSO). The final concentration of DMSO was less than $0.1 \%$.

\section{KARGER}


Cell culture and treatment

Murine RAW264.7 macrophages, obtained from the American Type Culture Collection (Rockville, MD), were cultured in DMEM containing $10 \% \mathrm{FBS}, 100 \mathrm{U} / \mathrm{ml}$ penicillin, and $100 \mu \mathrm{g} / \mathrm{ml} \mathrm{streptomycin} \mathrm{in}$ $5 \% \mathrm{CO}_{2}$ at $37^{\circ} \mathrm{C}$. In some experiments, the cells were preincubated with ZY-1A4 $(12.5-50 \mu \mathrm{M}, 4 \mathrm{~h}), \mathrm{CORM}-3$ $(100 \mu \mathrm{M}, 1 \mathrm{~h})$, bilirubin $(20 \mu \mathrm{M}, 1 \mathrm{~h}), \mathrm{ZnPP}(\mathrm{a}$ HO-1 inhibitor, $10 \mu \mathrm{M}, 1 \mathrm{~h})$, SB203580 (a p38 inhibitor, $10 \mu \mathrm{M}$, 1h), LY294002 (a PI3K inhibitor, $10 \mu \mathrm{M}, 1 \mathrm{~h}$ ), Wortmannin (a PI3K inhibitor, $1 \mu \mathrm{M}, 1 \mathrm{~h}$ ), or SP60012 (a JNK1/2 inhibitor, $10 \mu \mathrm{M}, 1 \mathrm{~h})$, then stimulated with or without LPS $(1 \mu \mathrm{g} / \mathrm{ml})$ for indicated periods to measure MAPK (p38, ERK1/2, and JNK1/2) phosphorylation, NF-KB p65 nuclear translocation, NF- $\mathrm{kB}$ binding activity, nitric oxide (NO) release, inducible nitric oxide synthase (iNOS) and HO-1 expression as well as cell viability.

\section{Cell viability assay}

Cell viability was evaluated by MTT assay. In brief, RAW264.7 cells in 96-well plates were incubated with different concentrations of ZY-1A4 for $24 \mathrm{~h}$, the medium was changed before the assay. MTT dissolved in phosphate buffered saline (PBS), was added to the culture media to reach a final concentration of $0.5 \mathrm{mg} /$ $\mathrm{ml}$. After incubation at $37^{\circ} \mathrm{C}$ for $4 \mathrm{~h}$, the culture media containing MTT was removed. DMSO was then added into each well, and the absorbance at $570 \mathrm{~nm}$ was measured using a microplate reader (M1000, TECAN, Austria GmbH, Austria).

\section{NO production assay}

RAW 264.7 cells in 12-well plates were incubated with different concentrations of ZY-1A4 or other treatment. Subsequently, stimulated with or without LPS $(1 \mu \mathrm{g} / \mathrm{ml})$ for $24 \mathrm{~h}$, NO production in the medium was assessed by measuring nitrite/nitrate using the NO detection Kit (Beyotime Biotechnology, Jiangsu, China) according to the manufacturer's instructions. The absorbance of the mixture at $540 \mathrm{~nm}$ was determined using a microplate reader (M1000, TECAN, Austria GmbH, Austria), and nitrite concentration was determined using a dilution of sodium nitrite as a standard.

\section{siRNA transfection}

RAW264.7 cells were transfected with mouse HO-1 siRNA (sc-35555), Keap1 siRNA (sc-43879), or FITC-conjugate scrambled siRNA (sc-36869, all siRNA were from Santa Cruz Biotechnology) by using Lipofectamine RNAiMAX and Opti-MEM medium (all from Life Technologies, Shanghai, China). Briefly, RAW264.7 cells were plated on 12 -well plates at $30 \%$ to $50 \%$ confluence $24 \mathrm{~h}$ before transfection. Individual siRNAs (at 25 to $50 \mathrm{nM}$ ), Lipofectamine RNAiMAX, and Opti-MEM were mixed and incubated at room temperature for $20 \mathrm{~min}$. siRNA-oligofectamine complexes were added to cells for $24 \mathrm{~h}$ and the medium was replaced by fresh serum free DMEM medium after transfection for 72 h. Knockdown of HO-1 or Keap1 was assessed by Western blot.

\section{Preparation of whole cell extracts and nuclear fraction}

For whole cell extraction, cells were washed twice with ice-cold PBS and lyzed in RIPA buffer with protease \& phosphatase inhibitor. After centrifugation $\left(4^{\circ} \mathrm{C}, 10 \mathrm{~min}, 10,000 \mathrm{~g}\right)$, samples were prepared for Western blot analysis.

For preparation of nuclear fraction, nuclear proteins of RAW264.7 cells were extracted using the NEPER $^{\circledR}$ Nuclear and Cytoplasmic Extraction Reagents (ThermoFisher Scientific, Shanghai, China) according to manufacturer's instructions.

\section{Western blot assay}

Western blot analyses was performed as previously described [8]. Equal amounts $(50 \mu \mathrm{g})$ of proteins were separated and transferred to polyvinyl difluoride membrane. After blocked with $5 \%$ non-fat dried milk, the membranes were probed with antibodies: p-ERK1/2, p-JNK1/2, p-p38, ERK1/2, JNK1/2, p38, p65 (all dilution in 1:1000), iNOS (1:500), $\beta$-actin (1:2000), Keap1 (1:500), Nrf2 (1:500), HO-1 (1:500), or Lamin A/C (1:3000) and incubation with either horseradish peroxidase-conjugated goat anti-rabbit or anti-mouse antibody (1:5000, ThermoFisher Scientific, Shanghai, China). Immunoreactive proteins were visualized by enhanced chemiluminescence and signal intensity was detected and quantified by Alpha Imager (Alpha Innotech Corp, San Leandro, CA).

\section{KARGER}




\section{$N F-\kappa B$ activity assay}

The nuclear extracts $(10 \mu \mathrm{g})$ from RAW 264.7 cells were analyzed in duplicate for NF- $\kappa$ B DNAbinding activity using TransAM ${ }^{\mathrm{TM}}$ NF- $\mathrm{KB}$ p65 assay kit (40096, Active Motif) following the manufacturer's instructions. The $\mathrm{OD}_{450}$ was measured by a microplate reader (M1000, TECAN, Austria GmbH, Austria).

Electrophoretic mobility shift assay (EMSA)

EMSA was applied to determine NF- $\kappa$ B DNA-binding activity by using the LightShift Chemiluminescent EMSA Kit (ThermoFisher Scientific, Shanghai, China) according to the manufacturer's instructions. Briefly, nuclear protein $(3 \mu \mathrm{g})$ was incubated at room temperature for $30 \mathrm{~min}$ with biotin-labeled probes and poly $(\mathrm{dI}-\mathrm{dC})$ as an inhibitor of nonspecific binding. After electrophoresis on $6 \%$ nondenaturing polyacrylamide gels, the samples on the gel were transferred to a positive charge nylon membrane. After the transfer was completed, the membrane was cross-linked and biotin-labeled DNA was detected by using a chemiluminescent detection kit (Pierce, Rockford, IL).

\section{Statistical analysis}

Results of the experimental studies were expressed as mean \pm standard error of the mean (SEM). All data analysis was performed with the use of GraphPad Prism 5 software. Differences between mean values of multiple groups were analyzed by one-way analysis of variance with Tukey's test for post hoc comparisons. Statistical significance was considered at $P<0.05$.

\section{Results}

\section{ZY-1A4 inhibited LPS-induced NO production and iNOS expression}

To demonstrate the anti-inflammatory activity of ZY-1A4, RAW264.7 cells were treated with indicated concentrations of ZY-1A4 for $4 \mathrm{~h}$ before exposure to LPS ( $1 \mu \mathrm{g} / \mathrm{mL})$ for 24 h. As shown in Fig. 1C, LPS stimulation markedly enhanced NO production, which was concentration-dependently attenuated by ZY-1A4 $(P<0.05)$. The associated iNOS expression was also suppressed by ZY-1A4 in a concentration dependent manner $(P<0.05$, Fig. 1D). However, the other compounds derived from sodium 9-hydroxyltanshinone IIA sulfonate did not show significant inhibitory effect on LPS-induced NO production (data not shown). Further, ZY-1A4 (12.5-200 $\mu \mathrm{M})$ alone didn't show cytotoxicity for 24 in RAW264.7 cells as checked by MTT assay $(P>0.05$, Fig. 1E), suggesting that no-cytotoxic effect of ZY-1A4 was observed at the concentrations used in this study. Altogether, the data suggested that ZY-1A4 could suppress iNOS expression and NO production induced by LPS.

\section{ZY-1A4 suppressed LPS-activated JNK1/2 signaling}

In activated-macrophages, MAPK (JNK1/2, p38, and ERK1/2) play pivotal role in signaling pathways that control the synthesis and release of inflammatory mediators and cytokines [24, 25]. To determine whether the inhibition of iNOS expression was mediated through the MAPK pathway, we examined the effect of ZY-1A4 on LPS-induced phosphorylation of ERK1/2, JNK1/2, and p38 MAPK in RAW264.7 cells. As shown in Fig. 2A, B, and C, ZY-1A4 also inhibited LPS-induced phosphorylation of JNK1/2, but not p38 or ERK1/2, in concentration dependent manner $(P<0.05)$. In addition, LPS time-dependently induced phosphorylation of ERK1/2, JNK1/2, and p38. Interestingly, ZY-1A4 (50 $\mu \mathrm{M})$ timedependently inhibited LPS-induced JNK1/2 phosphorylation without any effect on total JNK1/2 expression $(P<0.05$, Fig. 2D). However, ZY-1A4 didn't suppress phosphorylation of ERK1/2 or p38 induced by LPS $(P>0.05$, Fig. $2 \mathrm{E}$ and F). These results strongly indicated that ZY-1A4 inhibited LPS-mediated activation of JNK1/2 signaling, which might be involved in the inhibition of inflammatory responses in LPS-stimulated RAW 264.7 cells.

\section{ZY-1A4 modulated LPS-induced NF- $\kappa B$ activation}

Activated NF- $\kappa$ B regulates iNOS expression and plays an important role in inflammation in LPS-stimulated macrophages [26, 27]. In present study, LPS stimulation for $2 \mathrm{~h}$ caused 
A<smiles>Cc1c(S(=O)(=O)O[Na])oc2c3c(ccc12)-c1ccc2c(c1C(=O)C3=O)CCC(C)(C)C2(C)C</smiles>

STS

B<smiles></smiles>

ZY-1A4

D
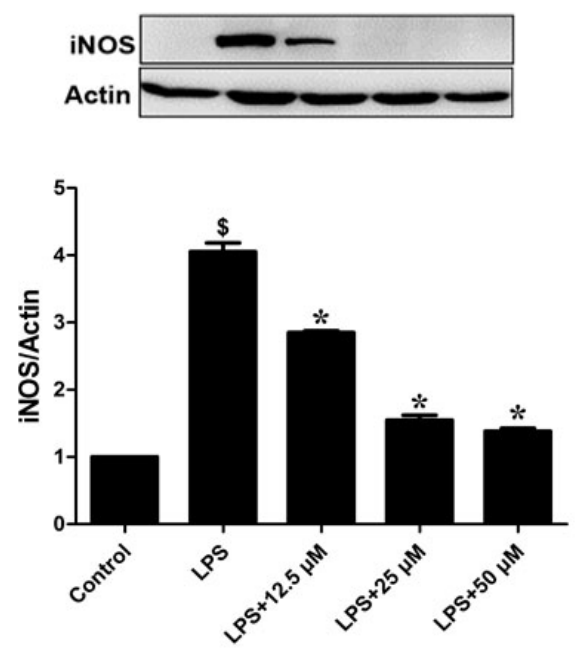

C

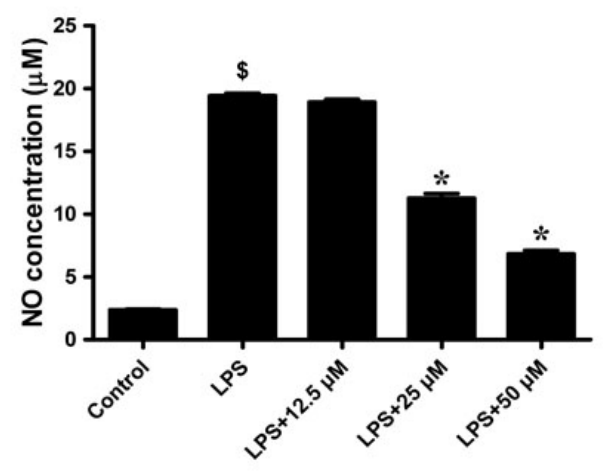

E

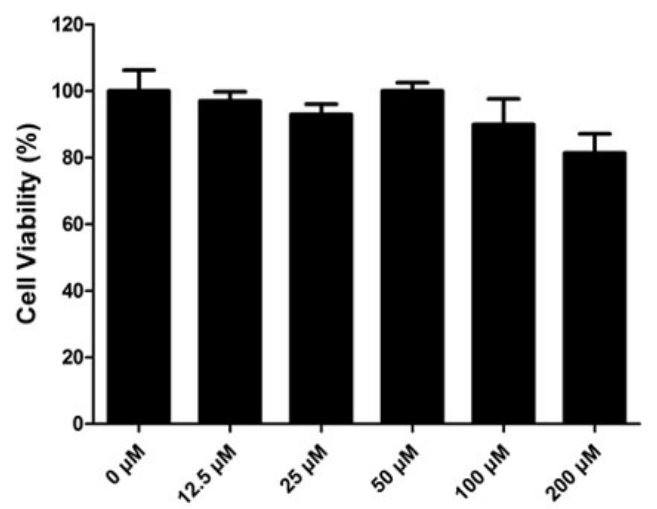

Fig. 1. ZY-1A4 inhibited LPS-induced NO production and iNOS expression. The chemical structures of sodium tanshinone IIA sulfonate (STS, A) and sodium 9-acetoxyltanshinone IIA sulfonate (ZY-1A4, B). Cells were treated with indicated concentrations of ZY-1A4 for $4 \mathrm{~h}$, then stimulated with or without LPS (1 $\mu \mathrm{g} / \mathrm{ml})$ for $24 \mathrm{~h}$, NO production (C), iNOS expression (D), and cell viability (E) were assessed by assays as described in Materials and Methods, respectively $(n=9)$; $\beta$-actin was used as loading control. Protein expression in control cells was set to $1(n=9) ;{ }^{\$} P<0.05$ compared with unstimulated cells, ${ }^{*} P<0.05$ compared with LPS-stimulated cells.

a significant nuclear translocation of NF- $\mathrm{BB}$ p65, which was concentration-dependently attenuated by ZY-1A4 $(P<0.05$, Fig. $3 \mathrm{~A})$. In addition, ZY-1A4 concentration-dependently inhibited LPS-induced binding activity of NF- $\kappa B$ to DNA oligonucleotides by using ELISAbased DNA binding analysis $(P<0.05$, Fig. 3B). The similar profile on LPS-induced binding activity of NF- $\kappa \mathrm{B}$ was also further confirmed by EMSA $(P<0.05$, Fig. $3 \mathrm{C})$. Taken together, these results indicated that inhibiting NF- $\mathrm{BB}$ activation by ZY-1A4 might be responsible for suppressing NO release and iNOS expression in LPS-stimulated RAW264.7 cells.

\section{ZY-1A4 induced HO-1 expression involvement of PI3K/Akt signaling}

We previously reported that a derivative of danshensu, another active compound of danshen, was able to induce the expression of HO-1 [12]. To determine the effect of ZY1A4 on HO-1 expression, cells were incubated with various concentrations of ZY-1A4 for 


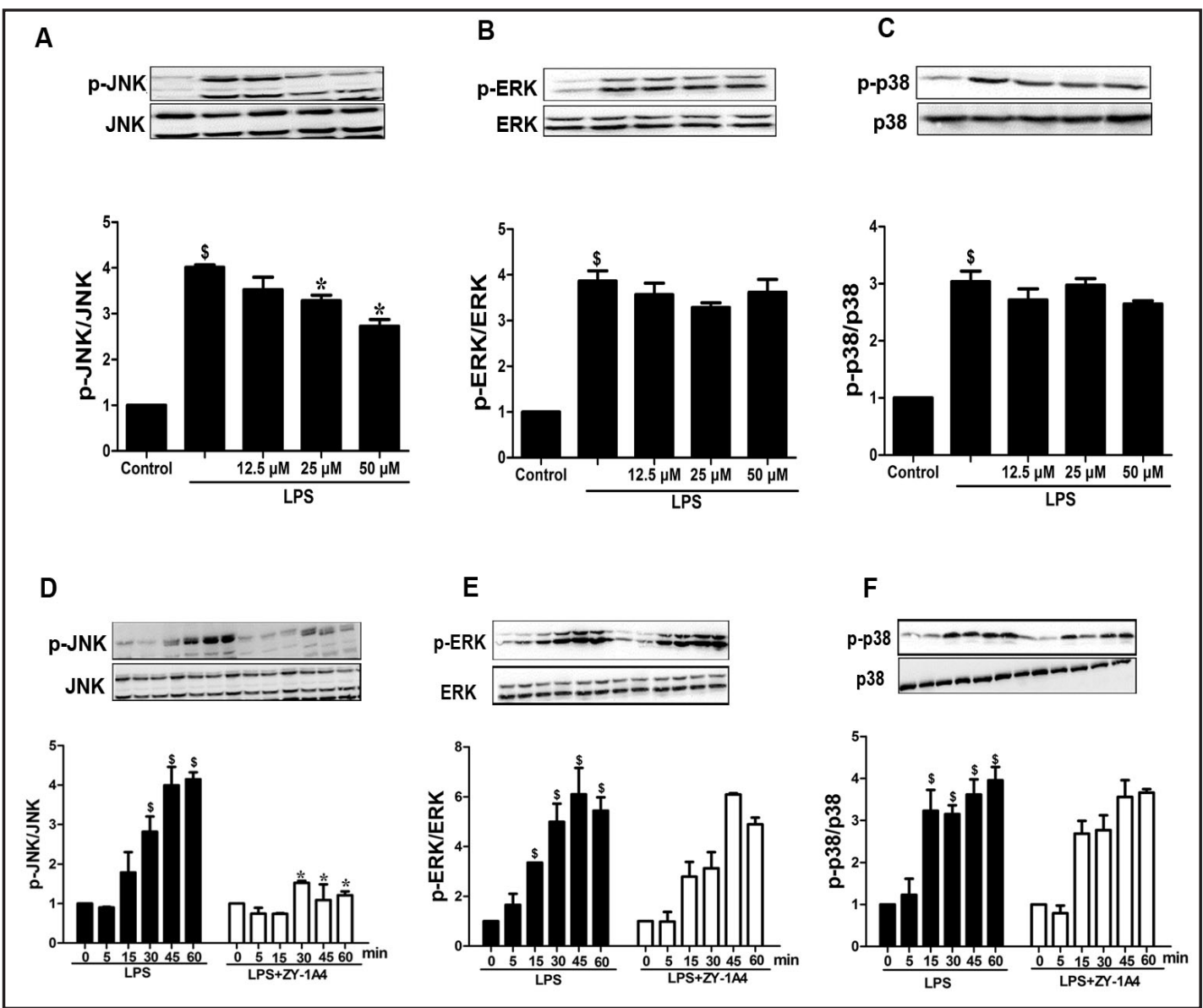

Fig. 2. ZY-1A4 concentration- and time-dependently suppressed LPS-activated JNK1/2 signaling. Cells were treated with indicated concentrations of ZY-1A4 for $4 \mathrm{~h}$, then stimulated with or without LPS (1 $\mu \mathrm{g} / \mathrm{ml})$ for $45 \mathrm{~min}$, Western blot and quantitative analysis for p-JNK1/2/JNK1/2 (A), p-ERK1/2/ERK1/2 (B), and p-p38/p38 (C); Cells were treated with ZY-1A4 (50 $\mu \mathrm{M})$ for $4 \mathrm{~h}$, then stimulated with LPS $(1 \mu \mathrm{g} / \mathrm{ml})$ for indicated periods, Western blot and quantitative analysis for p-JNK1/2/JNK1/2 (D), p-ERK1/2/ERK1/2 (E), and p-p38/p38 (F). Protein expression in control cells was set to $1(n=9)$. ${ }^{\$} P<0.05$ compared with unstimulated cells, ${ }^{*} P<0.05$ compared with LPS-stimulated cells.

the indicated periods. Western blot analysis indicated that ZY-1A4 markedly induced HO-1 expression in a concentration- and time-dependent manner, with a maximal response at a concentration of $100 \mu \mathrm{M}$ for $12 \mathrm{~h}$, and maintaining up to $24 \mathrm{~h}$ (Fig. $4 \mathrm{~A}$ and B).

To elucidate the roles of MAPK and PI3K/Akt signaling in ZY-1A4-mediated HO-1 expression, cells were pretreated with LY294002, SP60012, or SB203580. As shown in Fig. 4C, LY294002, but not SP60012 or SB203580, significantly inhibited ZY-1A4-mediated HO-1 expression $(P<0.05)$. In addition, ZY-1A4, but not SB203580, SP60012, or LY294002, also enhanced HO-1 expression in LPS-stimulated RAW264.7 cells $(P<0.05)$. LPS tended to induce HO-1 expression, but this increase was not statistically significant $(P>0.05$, Fig. 4D). To further confirm whether PI3K/Akt signaling was involved in ZY-1A4-mediated HO-1 protein. Indeed, as shown by the detection of the phosphorylation of Akt (Fig. 4E and F), ZY1A4 markedly induced Akt phosphorylation in a concentration- and time-dependent manner. In addition, the pharmacological PI3K inhibitor LY294002 or Wortmannin abrogated the ZY1A4-induced the phosphorylation of Akt (Fig. 4G). Collectively, our results suggested that ZY-1A4-induced HO-1 expression was mediated through a PI3K/Akt-dependent signaling pathway. 


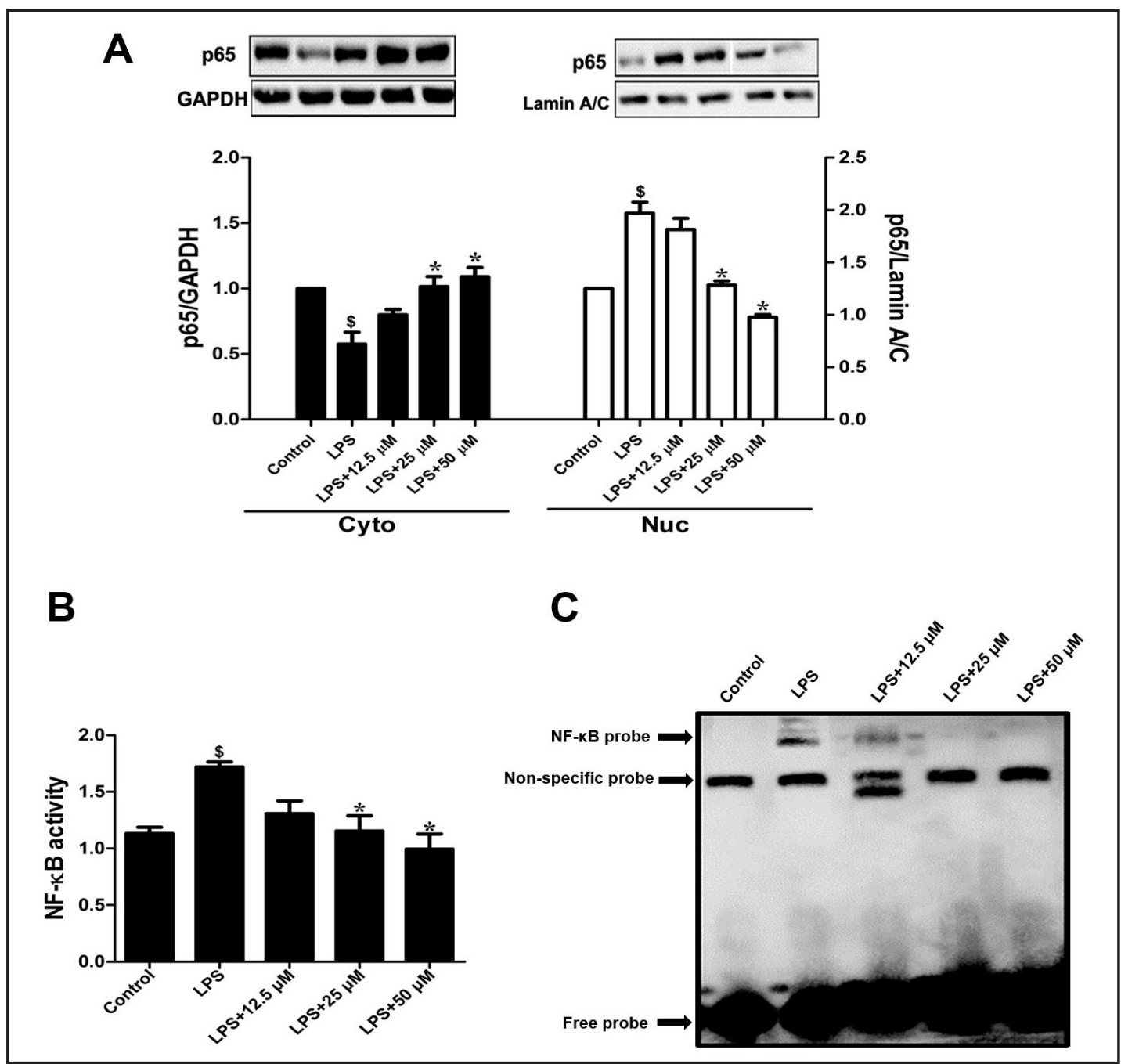

Fig. 3. ZY-1A4 modulated LPS-induced NF- $\kappa B$ activation. Cells were treated with indicated concentration of $\mathrm{ZY}-1 \mathrm{~A} 4$, then stimulated with or without LPS $(1 \mu \mathrm{g} / \mathrm{ml})$ for $2 \mathrm{~h},(\mathrm{~A})$ Western blot and densitometric analysis for level of p65 in cytoplasmic and nuclear fraction, GAPDH and Lamin A/C were used as loading control for cytoplasmic and nuclear fraction, respectively. Protein expression in control cells was set to $1(n=9)$. (B) NF- $\mathrm{B}$ p 65 DNA binding activity was measured by using ELISA-based DNA binding analysis $(n=9)$; (C) NF$\kappa \mathrm{B}$ p65 DNA binding activity was measured by EMSA. ${ }^{\$} P<0.05$ compared with unstimulated cells, ${ }^{*} P<0.05$ compared with LPS-stimulated cells.

Modulation of Keap1/Nrf2 signaling mediated ZY-1A4-induced HO-1 expression

To further investigate the possible mechanism, we explored whether ZY-1A4 modulate Keap1/Nrf2 signaling. Western blot analyses revealed that ZY-1A4 downregulated Keap1 in cells with increasing periods (Fig. 5A). Simultaneously, level of Nrf2 protein in the nucleus was also increased gradually over time (Fig. 5B). We postulated that ZY-1A4 might induce degradation of Keap 1 which explained the reduced levels of Keap1 with concomitant increase in nuclear Nrf2. As shown in Fig. 5C, Keap1 siRNA, but not scrambled siRNA, resulted in significant reduction of Keap1 expression $(P<0.05)$. We also found that ZY-1A4 $(50 \mu \mathrm{M})$ could mimic Keap1 siRNA knockdown-mediated Nrf2 nuclear accumulation. Moreover, inhibition of PI3K/Akt signaling by LY294002 blocked ZY-1A4-mediated Nrf2 nuclear translocation $(P<0.05$, Fig. 5D). Taken together, these results suggested that the upregulation of HO- 1 by ZY-1A4 was mediated through activation of PI3K/Akt-Nrf2 pathway.

\section{KARGER}


A
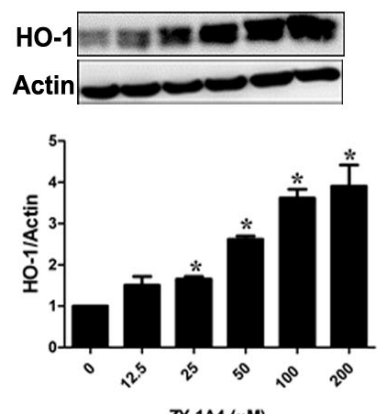

C
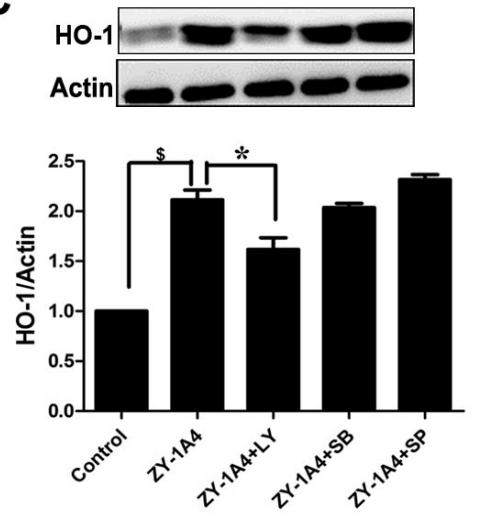

B
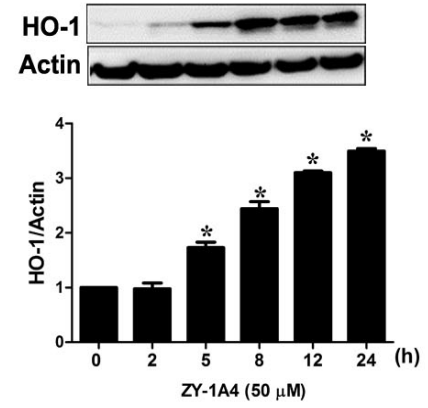

D
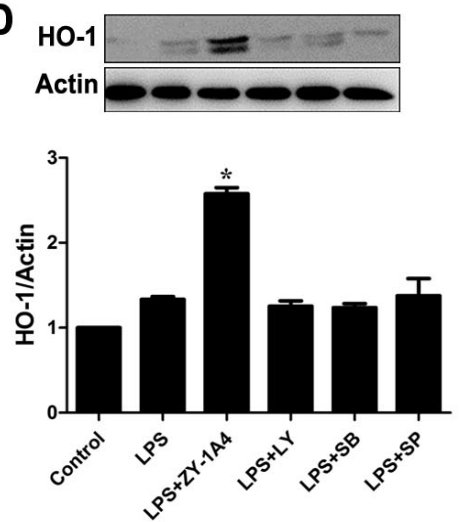

E
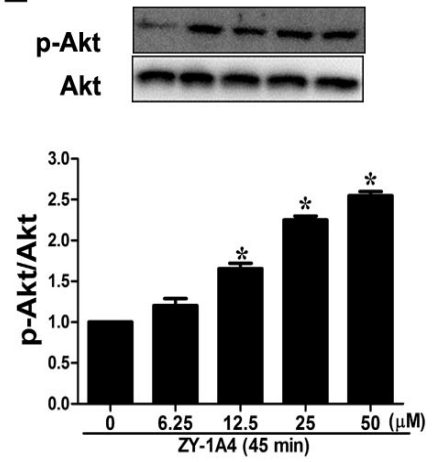

$\mathbf{F}$
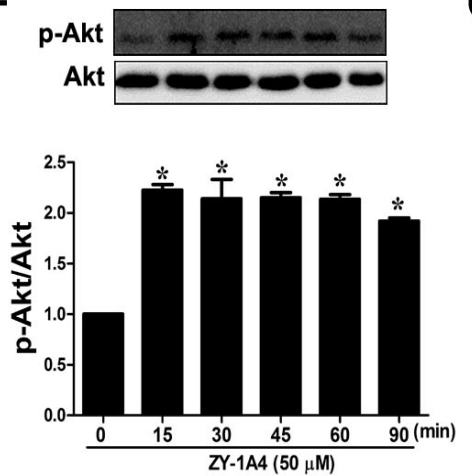

G
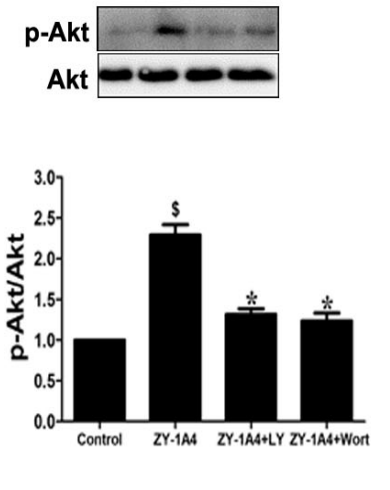

Fig. 4. ZY-1A4 induced HO-1 expression via activating of PI3K/Akt signaling. (A) Cells were incubated with indicated concentrations of ZY-1A4 for $12 \mathrm{~h}$. (B) Cells were incubated with ZY-1A4 (50 $\mu \mathrm{M})$ for indicated periods. (C) Cells treated with LY294002 (LY, $10 \mu \mathrm{M})$, SB203580 (SB, $10 \mu \mathrm{M})$, or SP60012 (SP, $10 \mu \mathrm{M})$ for $1 \mathrm{~h}$, then incubated with ZY-1A4 (50 $\mu \mathrm{M})$ for $12 \mathrm{~h}$. (D) Cells treated with SB, SP, LY, or ZY-1A4 (50 $\mu \mathrm{M}, 4 \mathrm{~h})$, then stimulated with or with LPS for $12 \mathrm{~h}$. (A) (B) (C) (D): Western blot and quantitative analysis for HO-1 expression, $\beta$-actin was used as loading control. (E) Cells were incubated with indicated concentrations of ZY-1A4 for 45 min. (G) Cells were incubated with ZY-1A4 $(50 \mu \mathrm{M})$ for indicated periods. (F) Cells treated with LY or Wortmannin (Wort, $1 \mu \mathrm{M}$ ) for $1 \mathrm{~h}$, then incubated with ZY-1A4 (50 $\mu \mathrm{M}$ ) for $45 \mathrm{~min}$. (E) (F) (G): Western blot and quantitative analysis for p-Akt expression, Akt was used as loading control; Protein expression in control cells was set to $1(n=9)$. ${ }^{*} P<0.05$ compared with unstimulated cells, ${ }^{\$} P<0.05$ compared with ZY-1A4-treated cells.

Upregulation of $\mathrm{HO}-1$ by $\mathrm{ZY}-1 \mathrm{~A} 4$ contributed to its anti-inflammatory activity

To determine whether anti-inflammatory activities of ZY-1A4 was mediated through the induction of HO-1 expression, the role of ZY-1A4-mediated HO-1 in LPS-stimulated 


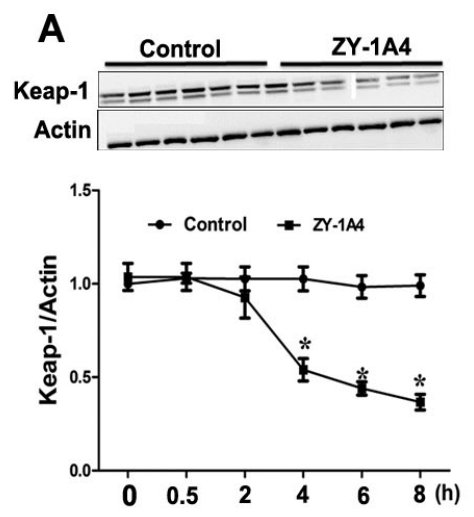

C

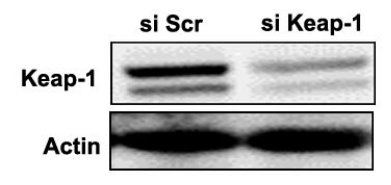

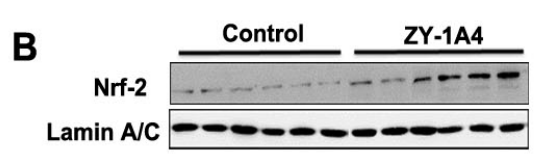

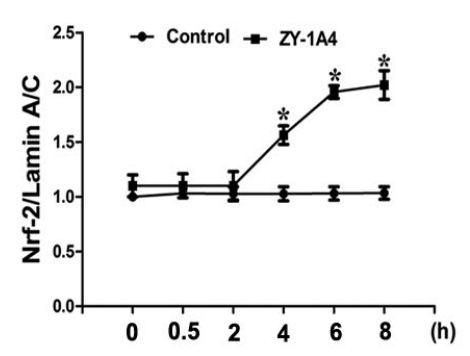

D
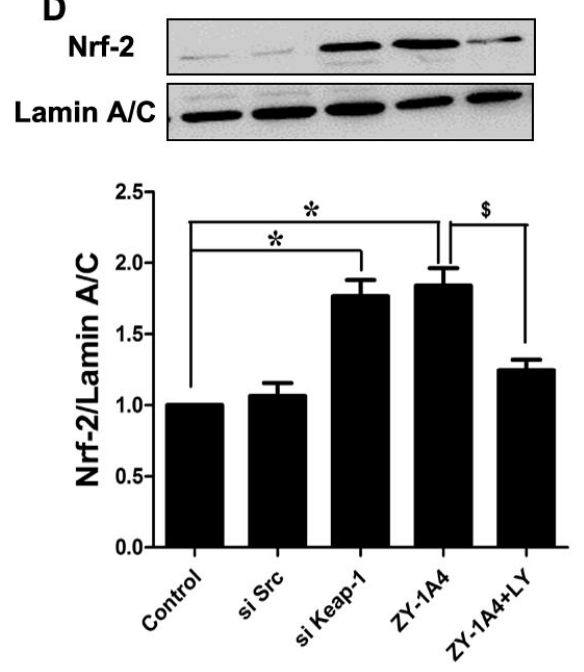

Fig. 5. Modulation of Keap1/Nrf2 signaling mediated ZY-1A4-induced HO-1 expression. Cells were incubated with ZY-1A4 $(50 \mu \mathrm{M})$ for indicated periods, Western blot and densitometric analysis level of Keap1 (A) and Nrf2 (B) in cytoplasmic and nuclear fraction, GAPDH and Lamin A/C were used as loading control for cytoplasmic and nuclear fraction, respectively. (C) Cells were transfected with Keap1 siRNA or scrambled siRNA, representative photomicrographs of Keap1 expression were detected by Western blot, $\beta$-actin was used as loading control; (D) Cells were treated with keap1 siRNA, scrambled siRNA, or LY with or without ZY-1A4 for $4 \mathrm{~h}$, Western blot and densitometric analysis level of Nrf2 in nuclear fraction, Lamin A/C was used as loading control for nuclear fraction. Protein expression in control cells was set to $1(n=9)$. ${ }^{*} P<0.05$ compared with unstimulated cells, ${ }^{\$} P<0.05$ compared with ZY-1A4-treated cells.

macrophages was analyzed by specific inhibitors or knockdown strategy. As shown in Fig. $6 \mathrm{~A}$ and $\mathrm{B}, \mathrm{ZY}-1 \mathrm{~A} 4(25 \mu \mathrm{M})$ significantly diminished LPS-stimulated iNOS expression and NO production in the macrophages $(P<0.05)$. Meanwhile, LY294002, SP60012, and SB203580 all markedly suppressed LPS-driven iNOS expression and NO production $(P<0.05)$. In addition, ZY-1A4 and bilirubin, but not CORM-3, markedly induced HO-1 expression in LPS-stimulated cells $(P<0.05)$. LY294002, but not ZnPP, significantly decreased ZY-1A4mediated HO-1 expression $(P<0.05$, Fig. 6C). ZnPP, but not LY294002, significantly reversed the inhibitory effects of ZY-1A4 on LPS-induced NO production and iNOS expression $(P<0.05)$. The HO-1 by-product CO (CORM-3, a CO donor) or bilirubin could mimic the effects of ZY-1A4 on LPS-induced NO production and iNOS expression $(P<0.05$, Fig. 6D 


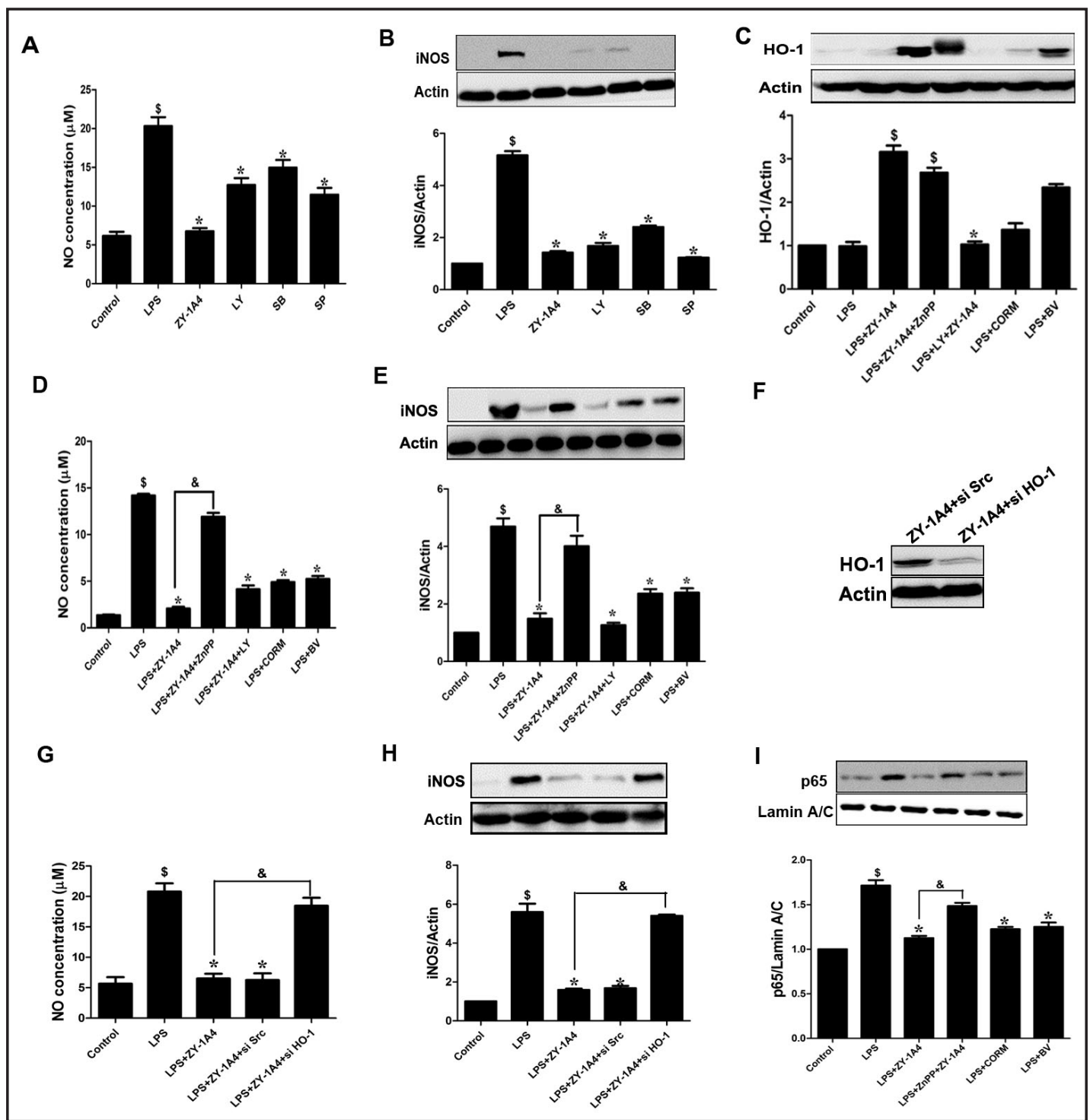

Fig.6. Upregulation of HO-1 by ZY-1A4 contributed to its anti-inflammatory activity. Cells treated with SB, SP, LY, or ZY-1A4 (50 $\mu \mathrm{M}, 4 \mathrm{~h})$, then stimulated with or without LPS for $24 \mathrm{~h},(\mathrm{~A})$ NO production was assessed by using the NO detection Kit (n=9); (B) Western blot and quantitative analysis for iNOS expression, $\beta$-actin was used as loading control. Cells were pretreated with ZnPP $(10 \mu \mathrm{M})$ or LY for $1 \mathrm{~h}$, then incubated with ZY-1A4 $(50 \mu \mathrm{M})$, CORM-3, or bilirubin for $4 \mathrm{~h}$, further stimulated with or without LPS for another $24 \mathrm{~h}$. (C) Western blot and densitometric analysis for HO-1 expression, $\beta$-actin was used as loading control. (D) Bar graph showed quantitative analysis of NO generation $(n=9)$; (E) Western blot and densitometric analysis for iNOS expression, $\beta$-actin was used as loading control. (F) Cells were transfected with HO-1 siRNA or scrambled siRNA, then ZY-1A4 (50 $\mu \mathrm{M})$ for $24 \mathrm{~h}$, the levels of HO-1 was detected by Western blot. Representative photomicrographs of Keap1 was detected by Western blot, $\beta$-actin was used as loading control; Cells were transfected with HO-1 siRNA or scrambled siRNA, then co-incubated with or without ZY-1A4 $(50 \mu \mathrm{M})$ for $4 \mathrm{~h}$, then stimulated with LPS for another $24 \mathrm{~h}$, (G) Bar graph showed quantitative analysis of NO generation ( $n=9)$; $(H)$ Western blot and densitometric analysis for iNOS expression, $\beta$-actin was used as loading control. Protein expression in control cells was set to $1(n=9)$; (I) Cells were pretreated with ZnPP $(10 \mu \mathrm{M})$ for $1 \mathrm{~h}$, then incubated with ZY-1A4 (50 $\mu \mathrm{M})$, CORM-3, or bilirubin for $12 \mathrm{~h}$, further stimulated with or without LPS for another $2 \mathrm{~h}$. Western blot and densitometric analysis for level of p65 in nuclear fraction, Lamin A/C was used as loading control. Protein expression in control cells was set to 1 ( $n=9$ ). ${ }^{\$} P<0.05$ compared with unstimulated cells, ${ }^{*} P<0.05$ compared with LPS-stimulated cells, ${ }^{\&} P<0.05$ compared with LPS-stimulated cells with ZY-1A4.

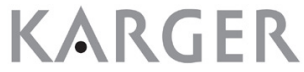


Fig. 7. Proposed working model by which ZY-1A4 regulated LPS-mediated inflammatory response in RAW264.7 cells. ZY-1A4 executed its anti-inflammatory activity in two ways-by down-regulating JNK1/2 signaling and inhibiting NF-KB activation that ultimately inhibitd the over expression of inflammation mediating enzymes; and by disrupting Nrf-2-Keap-1 complex leading to induction of cytoprotective enzymes that indirectly repress the expression of inflammatory mediators.

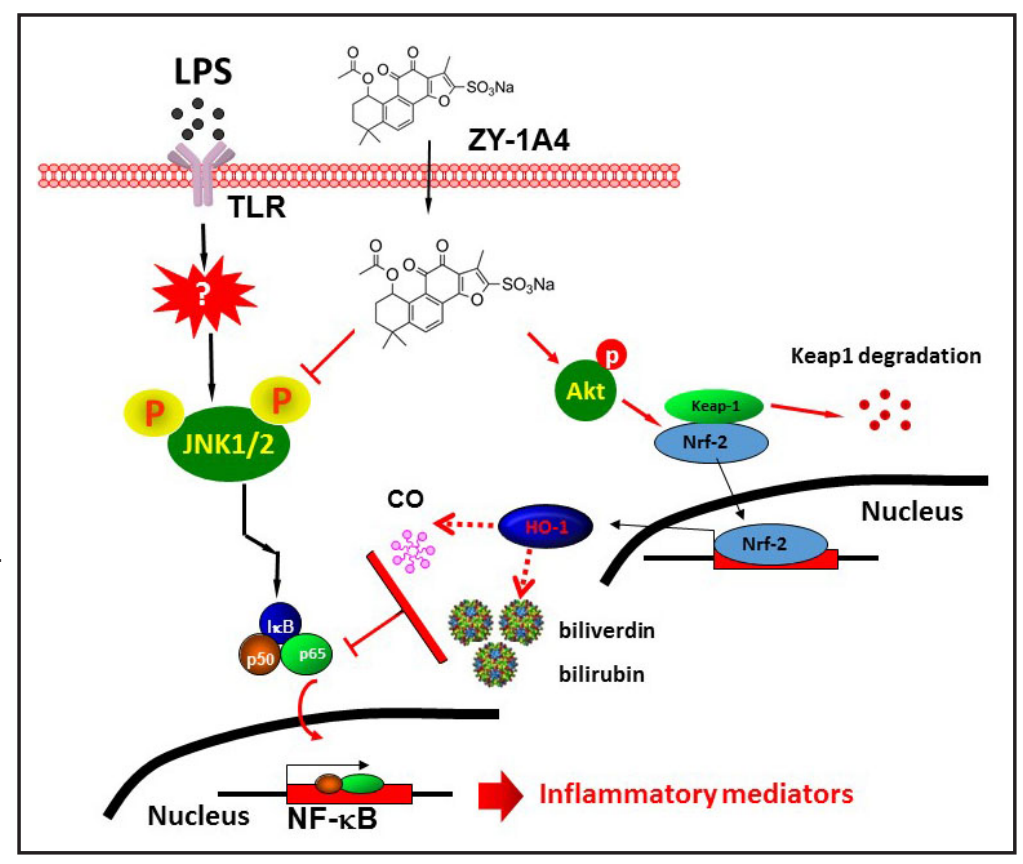

and E). Furthermore, knockdown of HO-1 using siRNA significantly reduced the ZY-1A4mediated HO-1 expression (Fig. 6F) and blocked the inhibitory effects of ZY-1A4 on LPSinduced NO production and iNOS expression $(P<0.05$, Fig. 6G and H). Furthermore, in order to test whether upregulation of $\mathrm{HO}-1$ by ZY-1A4 contributed to inhibit LPS-mediated NF- $\mathrm{BB}$ activation, the levels of NF- $\kappa B$ nuclear translocation p65 was analyzed by Western blot. As shown in Fig. 3I, LPS stimulation for $2 \mathrm{~h}$ induced a significant nuclear translocation of NF- $\kappa \mathrm{B}$ p65, which was markedly blocked by ZY-1A4, CORM-3 or bilirubin pretreatment $(P<0.05)$. However, inhibition of HO-1 using ZnPP significantly reversed the inhibitory effects of ZY$1 \mathrm{~A} 4$ on LPS-induced NF- $\kappa \mathrm{B}$ p65 nuclear translocation $(P<0.05)$. But ZY-1A4 alone didn't affect NF- $\kappa B$ p 65 nuclear translocation. In summary, these results supported the notion that HO-1 expression partly contributed to the inhibitory effects of ZY-1A4 on LPS-induced inflammatory response in RAW264.7 macrophage.

\section{Discussion}

In present study, we showed that a novel compound ZY-1A4 inhibited LPS-induced iNOS expression and NO production in RAW264.7 cells. Our data further demonstrated that ZY$1 \mathrm{~A} 4$ had inhibitory effects on LPS-induced inflammatory responses by blocking the NF- $\mathrm{B}$ signaling pathways and inducing $\mathrm{HO}-1$ expression in macrophages.

Herein, we reported that a novel compound ZY-1A4, derived from sodium 9-hydroxyltanshinone IIA sulfonate (a major impurity of STS), abrogated iNOS expression and NO production in LPS-stimulated macrophages. Macrophages activation is involved in the pathological process of various inflammation-related diseases [28]. During LPSmediated inflammatory responses in macrophages, activation of MAPK and PI3K/Akt signaling cascades are the principal signaling molecules leading to upregulation of iNOS expression and NO production $[8,29]$. In accordance with data from other groups, our results indicated that $\mathrm{p} 38$, JNK1/2, and PI3K/Akt signaling participated in LPS-induced iNOS expression and NO release by their specific inhibitors SB203580, SP60012, and LY294002, respectively. However, ZY-1A4 only suppressed LPS-mediated phosphorylation of JNK1/2, but had little effect on ERK1/2 or p38 phosphorylation, which was consistent with previous observations that specific JNK1/2 inhibition attenuated LPS-mediated iNOS expression and NO production in RAW264.7 cells $[30,31]$. The activation of NF- $\kappa B$ is one of the more 
well studied downstream components of the JNK1/2 signaling pathway [32, 33]. NF- $\kappa B$ is present in the cytosol with the inhibitor protein IкB family in unstimulated cells. Upon stimulation with LPS, the I $\kappa$ B proteins are phosphorylated and degraded, promoting NF- $\kappa B$ to translocate into nucleus and to initiate transcription of inflammatory gene, including iNOS $[8,10,34]$. As shown in Fig. 3, ZY-1A4 could inhibit LPS-induced NF- $\kappa B$ activation, as indicated by decreased nuclear translocation and DNA binding activity of NF- $\kappa B$. Taken together, our data suggested that inhibition of inflammatory mediators by ZY-1A4 was partially mediated through blockade of JNK1/2 signaling and NF- $\mathrm{BB}$ activation.

Recently, HO-1 is recognized to exhibit important immunomodulatory and antiinflammatory functions $[13,35,36]$. A growing body of evidences suggests that HO-1 expression exerts anti-inflammatory activities by producing three anti-inflammatory metabolites (i.e., CO, ferrous iron, and biliverdin) [13, 14, 37]. In terms of the ZY-1A4-evoked inhibition of iNOS expression, we showed for the first time that ZY-1A4 was able to induce HO-1 expression in macrophages and that the observed HO-1 induction was a feature linked to the inhibition of iNOS by ZY-1A4. This idea was supported by the results as following: (1) the blockage of HO-1 activity by ZnPP (a specific HO-1 inhibitor) or knockdown of HO-1 using a siRNA approach markedly abolished the inhibitory effects of ZY-1A4 on iNOS expression and NO production, and (2) LPS-mediated iNOS expression and NO production were also effectively reduced by application of HO-1 products $\mathrm{CO}$ (CORM-3, a CO-donor) and biliverdin. This findings were in line with many studies that link HO-1 to anti-inflammatory features $[13,14]$. In addition, our data showed that ZY-1A4 increased the activation of Nrf2 and expression of $\mathrm{HO}-1$, but decreased NF- $\mathrm{KB}$ activation and reduced iNOS expression. The question was whether ZY-1A4-mediated HO-1 expression was responsible for the NF- $\mathrm{BB}$ inhibition. Previous studies showed that upregulation of $\mathrm{HO}-1$ negatively regulates NF- $\mathrm{KB}$ activation and inflammatory gene expression [15-17, 38]. HO-1 also negatively regulates NF$\kappa \mathrm{B}$ activation through production of its metabolites such as $\mathrm{CO}$, bilirubin, and free iron [39, 40]. In agreement with this notion, our present results demonstrated that ZY-1A4 treatment increases HO-1 expression, while decreasing NF- $\mathrm{KB}$ nuclear translocation. This effect on $\mathrm{NF}-\kappa \mathrm{B}$ nuclear translocation was suppressed by $\mathrm{ZnPP}$ and mimicked by the end-products of HO-1 activity, i.e., bilirubin and CORM3. Therefore, induction of HO-1 might be, at least in part, responsible for the anti-inflammatory property of ZY-1A4 in LPS-stimulated RAW264.7 cells.

$\mathrm{Nrf} 2$ is an important transcriptional factor involved in the anti-inflammatory action of phytochemicals and is associated with the induction of HO-1 [20]. In the present study, ZY-1A4 markedly induced Nrf2 nuclear translocation and Keap1 degradation with a concomitant increase in HO-1 expression, and silencing Keap1 increased Nrf2 nuclear translocation and HO-1 expression. These findings suggested that activation of Nrf2 was involved in ZY-1A4-induced HO-1 expression. Nuclear translocation of Nrf2 requires the activation of the upstream signaling pathways, such as MAPK and PI3K/Akt [38, 41, 42]. In the present study, ZY-1A4-mediated degradation of Keap1 and nuclear translocation of Nfr2 were also abolished by pretreatment with LY294002. Meanwhile, we showed that ZY$1 \mathrm{~A} 4$ activated the phosphorylation of Akt in concentration and time dependent manners; however, by using specific inhibitors of JNK1/2, p38, and Akt, we demonstrated that only the PI3K/Akt pathway was involved in the induction of HO-1 expression by ZY-1A4. The results were consistent with previous reports that PI3K/Akt cascade is a key regulator of HO-1 expression $[40,43]$. However, the role of PI3K/Akt signaling activation depends on the experimental condition. In present study, treatment of ZY-1A4 alone induced Akt phosphorylation in cells. ZY-1A4-mediated cytoprotective action was not been blocked by LY294002 in LPS-stimulated RAW264.7 cells. On the contrary, LY294002 significantly reduced LPS-induced iNOS expression and NO generation, which was consistent with the previous studies that activation of PI3K/Akt pathway participated in LPS-induced inflammatory mediators $[43,44]$. Meanwhile, many chemopreventive phytochemicals exhibited simultaneous induction of Nrf2-regulated cytoprotective protein expression and inhibition of NF- $\kappa \mathrm{B}$-regulated inflammatory response via activation of PI3K/Akt signaling 
pathway $[12,40]$. This contradictory results were likely that phosphorylation of Akt was involved in ZY-1A4 induction of gene expression which was important to defend against LPSinduced deleterious effect through the PI3K/Akt pathway. However, the precise mechanisms of PI3K/Akt signaling activation remained to be clarified.

In conclusion, our data demonstrated that a novel 9-hydroxyltanshinone IIA derivative, ZY-1A4, was able to suppress LPS-induced iNOS expression and NO generation via inhibition of JNK1/2/NF-KB activation and upregulation of HO-1 expression. We further demonstrated that superinduction of HO-1 by ZY-1A4 might be associated with the suppression of LPSmediated iNOS expression and NO release via activation of PI3K/Akt and keap1/Nrf2 signaling (Fig. 7). These results suggested that the novel compound ZY-1A4 was a potential agent for the treatment of inflammation-related cardiovascular diseases.

\section{Acknowledgments}

This work was supported by grants from National Natural Science Foundation of China (No. 81470164; 81202528; 81330080), Shanghai Committee of Science and Technology of China (No. 14JC1401100), National Major Scientific and Technological Special Project (No. 2012ZX09103101-064), a key laboratory program of the Education Commission of Shanghai Municipality (No. ZDSYS14005).

\section{Disclosure Statement}

There are no conflicts of interest to declare.

\section{References}

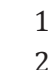

Medzhitov R: Origin and physiological roles of inflammation. Nature 2008;454:428-435.

Liu XH, Pan LL, Yang HB, Gong QH, Zhu YZ: Leonurine attenuates lipopolysaccharide-induced inflammatory responses in human endothelial cells: Involvement of reactive oxygen species and nf-kappab pathways. Eur J Pharmacol 2012;680:108-114.

Kondo T, Kawai T, Akira S: Dissecting negative regulation of toll-like receptor signaling. Trends Immunol 2012;33:449-458.

Mo C, Wang L, Zhang J, Numazawa S, Tang H, Tang X, Han X, Li J, Yang M, Wang Z, Wei D, Xiao H: The crosstalk between nrf2 and ampk signal pathways is important for the anti-inflammatory effect of berberine in lps-stimulated macrophages and endotoxin-shocked mice. Antioxid Redox Signal 2014;20:574-588.

Janeway CA, Jr., Medzhitov R: Innate immune recognition. Annu Rev Immunol 2002;20:197-216.

Xie S, Liu B, Fu S, Wang W, Yin Y, Li N, Chen W, Liu J, Liu D: Glp-2 suppresses lps-induced inflammation in macrophages by inhibiting erk phosphorylation and nf-kappab activation. Cell Physiol Biochem 2014;34:590-602.

Dong C, Davis RJ, Flavell RA: Map kinases in the immune response. Annu Rev Immunol 2002;20:55-72.

Liu XH, Pan LL, Jia YL, Wu D, Xiong QH, Wang Y, Zhu YZ: A novel compound dsc suppresses lipopolysaccharide-induced inflammatory responses by inhibition of akt/nf-kappab signalling in macrophages. Eur J Pharmacol 2013;708:8-13.

Pan LL, Liu XH, Gong QH, Wu D, Zhu YZ: Hydrogen sulfide attenuated tumor necrosis factor-alpha-induced inflammatory signaling and dysfunction in vascular endothelial cells. PLoS One 2011;6:e19766.

Jiang Q, Liu P, Wu X, Liu W, Shen X, Lan T, Xu S, Peng J, Xie X, Huang H: Berberine attenuates lipopolysaccharide-induced extracelluar matrix accumulation and inflammation in rat mesangial cells: Involvement of nf-kappab signaling pathway. Mol Cell Endocrinol 2011;331:34-40. 
11 Chen TH, Hsu YT, Chen CH, Kao SH, Lee HM: Tanshinone iia from salvia miltiorrhiza induces heme oxygenase-1 expression and inhibits lipopolysaccharide-induced nitric oxide expression in raw 264.7 cells. Mitochondrion 2007;7:101-105.

12 Pan LL, Liu XH, Jia YL, Wu D, Xiong QH, Gong QH, Wang Y, Zhu YZ: A novel compound derived from danshensu inhibits apoptosis via upregulation of heme oxygenase-1 expression in sh-sy5y cells. Biochim Biophys Acta 2013;1830:2861-2871.

13 Ryter SW, Alam J, Choi AM: Heme oxygenase-1/carbon monoxide: From basic science to therapeutic applications. Physiol Rev 2006;86:583-650.

-14 Sawle P, Foresti R, Mann BE, Johnson TR, Green CJ, Motterlini R: Carbon monoxide-releasing molecules (co-rms) attenuate the inflammatory response elicited by lipopolysaccharide in raw264.7 murine macrophages. Br J Pharmacol 2005;145:800-810.

15 Kim SJ, Lee SM: Nlrp3 inflammasome activation in d-galactosamine and lipopolysaccharide-induced acute liver failure: Role of heme oxygenase-1. Free Radic Biol Med 2013;65:997-1004.

16 Wei B, You MG, Ling JJ, Wei LL, Wang K, Li WW, Chen T, Du QM, Ji H: Regulation of antioxidant system, lipids and fatty acid beta-oxidation contributes to the cardioprotective effect of sodium tanshinone iia sulphonate in isoproterenol-induced myocardial infarction in rats. Atherosclerosis 2013;230:148-156.

17 Nakajima S, Kitamura M: Bidirectional regulation of nf-kappab by reactive oxygen species: A role of unfolded protein response. Free Radic Biol Med 2013;65:162-174.

18 Tao S, Zheng Y, Lau A, Jaramillo MC, Chau BT, Lantz RC, Wong PK, Wondrak GT, Zhang DD: Tanshinone i activates the nrf2-dependent antioxidant response and protects against as(iii)-induced lung inflammation in vitro and in vivo. Antioxid Redox Signal 2013;19:1647-1661.

19 Han JY, Fan JY, Horie Y, Miura S, Cui DH, Ishii H, Hibi T, Tsuneki H, Kimura I: Ameliorating effects of compounds derived from salvia miltiorrhiza root extract on microcirculatory disturbance and target organ injury by ischemia and reperfusion. Pharmacol Ther 2008;117:280-295.

20 Gao S, Liu Z, Li H, Little PJ, Liu P, Xu S: Cardiovascular actions and therapeutic potential of tanshinone iia. Atherosclerosis 2012;220:3-10.

21 Zhang K, Wang J, Jiang H, Xu X, Wang S, Zhang C, Li Z, Gong X, Lu W: Tanshinone iia inhibits lipopolysaccharide-induced muc1 overexpression in alveolar epithelial cells. Am J Physiol Cell Physiol 2014;306:C59-65.

22 Chen S, Zhao X, Li Y, Yang D, Zhou T, Fan G: Impurities preparation of sodium tanshinone iia sulfonate by high-speed counter-current chromatography and identification by liquid chromatography/multistage tandem mass spectrometry. J Chromatogr A 2013;1288:28-34.

23 Wang B, Wang H, Wang E, Yan W, Ye W, Li P: Isolation and structure characterization of related impurities in sodium tanshinone iia sulfonate by lc/esi-ms(n) and nmr. J Pharm Biomed Anal 2012;67-68:36-41.

24 Ho HJ, Huang DY, Ho FM, Lee LT, Lin WW: Inhibition of lipopolysaccharide-induced inducible nitric oxide synthase expression by endoplasmic reticulum stress. Cell Signal 2012;24:2166-2178.

25 Chen J, Liao MY, Gao XL, Zhong Q, Tang TT, Yu X, Liao YH, Cheng X: Il-17a induces pro-inflammatory cytokines production in macrophages via mapkinases, nf-kappab and ap-1. Cell Physiol Biochem 2013;32:1265-1274.

-26 Hadad N, Levy R: The synergistic anti-inflammatory effects of lycopene, lutein, beta-carotene, and carnosic acid combinations via redox-based inhibition of nf-kappab signaling. Free Radic Biol Med 2012;53:13811391.

27 Tak PP, Firestein GS: Nf-kappab: A key role in inflammatory diseases. J Clin Invest 2001;107:7-11.

28 Mosser DM, Edwards JP: Exploring the full spectrum of macrophage activation. Nat Rev Immunol 2008;8:958-969.

29 Beutler B: Inferences, questions and possibilities in toll-like receptor signalling. Nature 2004;430:257-263.

-30 You R, Long W, Lai Z, Sha L, Wu K, Yu X, Lai Y, Ji H, Huang Z, Zhang Y: Discovery of a potential antiinflammatory agent: 3-oxo-29-noroleana-1,9(11),12-trien-2,20-dicarbonitrile. J Med Chem 2013;56:19841995.

-31 Lin MW, Tsao LT, Chang LC, Chen YL, Huang LJ, Kuo SC, Tzeng CC, Lee MR, Wang JP: Inhibition of lipopolysaccharide-stimulated no production by a novel synthetic compound cyl-4d in raw 264.7 macrophages involving the blockade of mek4/jnk/ap-1 pathway. Biochem Pharmacol 2007;73:1796-1806. 


\section{Cellular Physiology Cell Physiol Biochem 2015;36:1316-1330 \begin{tabular}{ll|l} 
and Biochemistry 10.1159/000430299 & $\begin{array}{l}\text { O 2015 S. Karger AG, Basel } \\
\text { www.karger.com/cpb }\end{array}$ \\
\hline
\end{tabular} \\ Liu et al.: ZY-1A4 Inhibited Inflammation}

32 Maity B, Yadav SK, Patro BS, Tyagi M, Bandyopadhyay SK, Chattopadhyay S: Molecular mechanism of the anti-inflammatory activity of a natural diarylnonanoid, malabaricone c. Free Radic Biol Med 2012;52:16801691.

33 Pan Y, Wang Y, Cai L, Cai Y, Hu J, Yu C, Li J, Feng Z, Yang S, Li X, Liang G: Inhibition of high glucose-induced inflammatory response and macrophage infiltration by a novel curcumin derivative prevents renal injury in diabetic rats. Br J Pharmacol 2012;166:1169-1182.

-34 Brasier AR: The nuclear factor-kappab-interleukin-6 signalling pathway mediating vascular inflammation. Cardiovasc Res 2010;86:211-218.

35 Xue J, Habtezion A: Carbon monoxide-based therapy ameliorates acute pancreatitis via tlr4 inhibition. J Clin Invest 2014;124:437-447.

36 Chang CF, Liu XM, Peyton KJ, Durante W: Heme oxygenase-1 counteracts contrast media-induced endothelial cell dysfunction. Biochem Pharmacol 2014;87:303-311.

-37 Jamal Uddin M, Joe Y, Zheng M, Blackshear PJ, Ryter SW, Park JW, Chung HT: A functional link between heme oxygenase- 1 and tristetraprolin in the anti-inflammatory effects of nicotine. Free Radic Biol Med 2013;65:1331-1339.

-38 Wang J, Hu X, Xie J, Xu W, Jiang H: Beta-1-adrenergic receptors mediate nrf2-ho-1-hmgb1 axis regulation to attenuate hypoxia/reoxygenation-induced cardiomyocytes injury in vitro. Cell Physiol Biochem 2015;35:767-777.

-39 Bellezza I, Tucci A, Galli F, Grottelli S, Mierla AL, Pilolli F, Minelli A: Inhibition of nf-kappab nuclear translocation via ho-1 activation underlies alpha-tocopheryl succinate toxicity. J Nutr Biochem 2012;23:1583-1591.

40 Paine A, Eiz-Vesper B, Blasczyk R, Immenschuh S: Signaling to heme oxygenase-1 and its anti-inflammatory therapeutic potential. Biochem Pharmacol 2010;80:1895-1903.

-41 Wu BJ, Chen K, Barter PJ, Rye KA: Niacin inhibits vascular inflammation via the induction of heme oxygenase-1. Circulation 2012;125:150-158.

-42 Ma Q: Role of nrf2 in oxidative stress and toxicity. Annu Rev Pharmacol Toxicol 2013;53:401-426.

43 Park CM, Cho CW, Song YS: Top 1 and 2, polysaccharides from taraxacum officinale, inhibit nfkappabmediated inflammation and accelerate nrf2-induced antioxidative potential through the modulation of pi3k-akt signaling pathway in raw 264.7 cells. Food Chem Toxicol 2014;66:56-64.

44 Abarikwu SO: Kolaviron, a natural flavonoid from the seeds of garcinia kola, reduces lps-induced inflammation in macrophages by combined inhibition of il-6 secretion, and inflammatory transcription factors, erk1/2, nf-kappab, p38, akt, p-c-jun and jnk. Biochim Biophys Acta 2014;1840:2373-2381. 